\title{
Comparison of Monte Carlo Simulated k-ratios using WinX-Ray and WinCasino with Experimental Results
}

\author{
Philippe T.-Pinard and Raynald Gauvin
}

Department of Mining, Metals \& Materials Engineering, McGill University, 3610 University Street, Montréal, Québec, Canada, H3A 2B2

WinX-Rray ${ }^{1}$ and WinCasino ${ }^{2}$ are two softwares that simulate the trajectory and the interactions of electrons in a given material. To validate the exactitude of these softwares and to establish a point of comparison with other simulation softwares, over eight hundreds simulations were performed. These simulations come from a list of experimental k-ratios compiled by J.L. Pouchou ${ }^{3}$. They examine different binary alloys at different compositions and excitation energies ranging from $4 \mathrm{keV}$ to $30 \mathrm{keV}$. The experiments are designed to highlight the effect of the atomic number, the absorption and the borides compounds. Using WinX-Ray and WinCasino, Pouchou's database was simulated and k-ratios were calculated. The comparison between the simulated k-ratios and the experimental ones was performed by taking the quotient of the two. The result is a normal distribution centered around 1.0. Because of some limitations in the Monte Carlo simulation softwares, some experiments were discarded in the analysis. For instance, some simulated k-ratios were completely different from the Pouchou's k-ratios (ratio outside the $0.8-1.2$ brackets). These specific cases are later stated.

Figure 1 shows the results obtained with WinX-Ray (filled bars) and WinCasino (dashed bars). WinX-Ray has an average of 1.002161 and a standard deviation of $2.736 \%$ for 723 analyses out of 826. Simulations for copper $L_{\alpha}$, silver $L_{\beta}$ and uranium $M_{\beta}$ lines were not considered in the analysis. WinCasino has an average of 0.998986 and a standard deviation of $2.451 \%$ for 693 analyses out of 756 . The total number of simulations is less with WinCasino than with WinX-Ray because WinCasino does not generate $\beta$ lines. Also, simulations for copper $\mathrm{K}_{\alpha}$ and $\mathrm{L}_{\alpha}$ as well as boron $\mathrm{K}_{\alpha}$ were discarded. The latter problem with boron $\mathrm{K}_{\alpha}$ only applies for alloy with carbon and nitrogen. For these cases, the simulated k-ratios are much greater than the experimental k-ratios.

Figure 2, 3 and 4 illustrate, respectively, the results for the effect of the atomic number, the absorption and the borides compounds. The standard deviations are lower for each of these effects than for the overall standard deviation. Even if some experiments correspond to more than one group, results within a group are close to their average. For example, despite an average higher than 1.0 (simulated k-ratios are greater than experimental k-ratios), the standard deviation for the absorption effect is still low. WinCasino simulates very well the effect of boron when the experiment looks at the xrays of the other element in the alloy, but, as previously explained, has some difficulties to simulate X-rays for boron. This problem does not occur in WinX-Ray. In conclusion, WinX-Ray and WinCasino obtain similar results. WinCasino has smaller standard deviations but fewer simulations were considered in the analysis.

\footnotetext{
${ }^{1}$ R. Gauvin, E. Lifshin, H. Demers and P. Horny (2006), "WINX-Ray, A New Monte Carlo Program to Simulate the Complete EDS X-Ray Spectrum in the SEM", Microscopy \& Microanalysis, 12, pp. 1 - 16.

${ }^{2}$ P. Hovington, D. Drouin and R. Gauvin (1997), "Casino: A New Era of Monte Carlo Code in C Language for Electron Beam Interaction, Part I: Description of the Program", Scanning, Vol.19, pp. 1-14.

3 J.L. Pouchou and F. Pichoir (1991), "Quantitative Analysis of Homogeneous or Stratified Microvolumes applying the Model PAP”, in: Electron Probe Quantitation, Plenum Press, New York, pp. 31-75.
} 
Figure 1: All Analyses

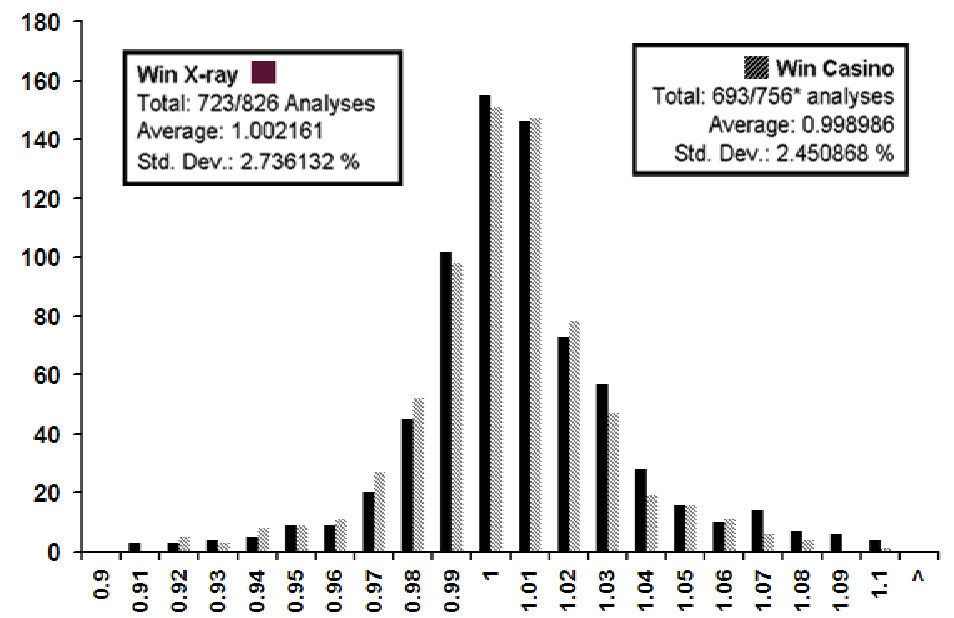

Figure 3: Absorption Effect

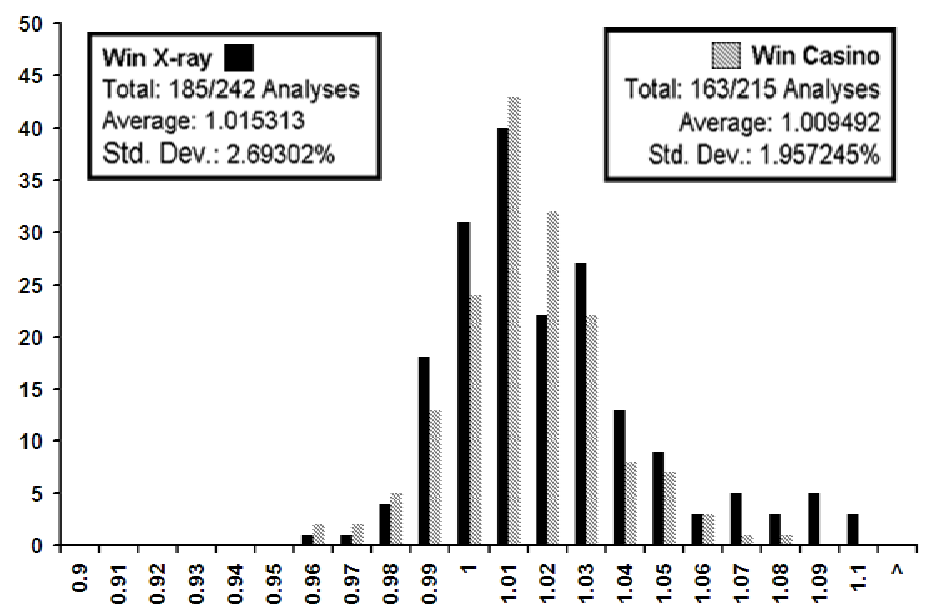

Figure 2: Atomic Number Effect

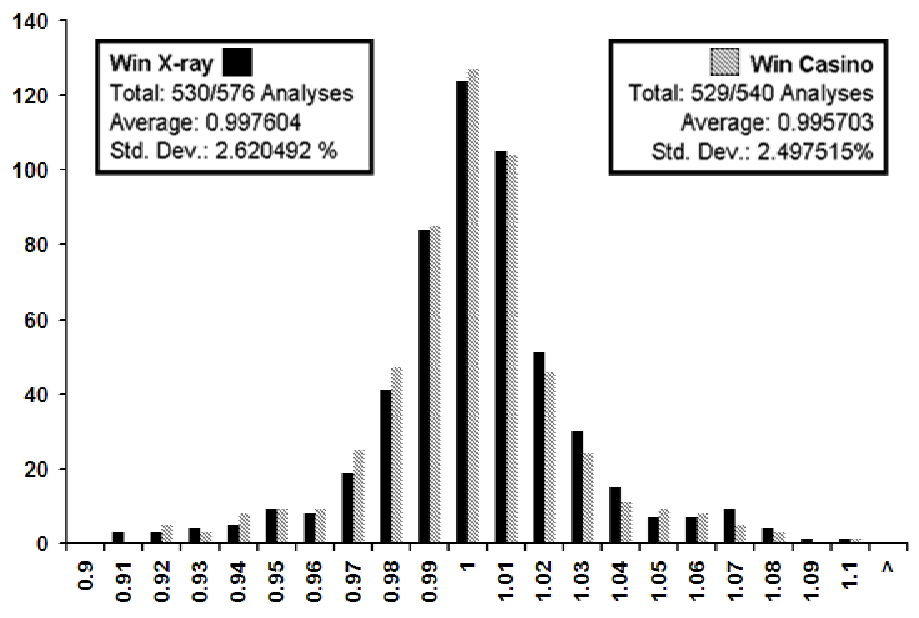

Figure 4: Borides Compounds Effect

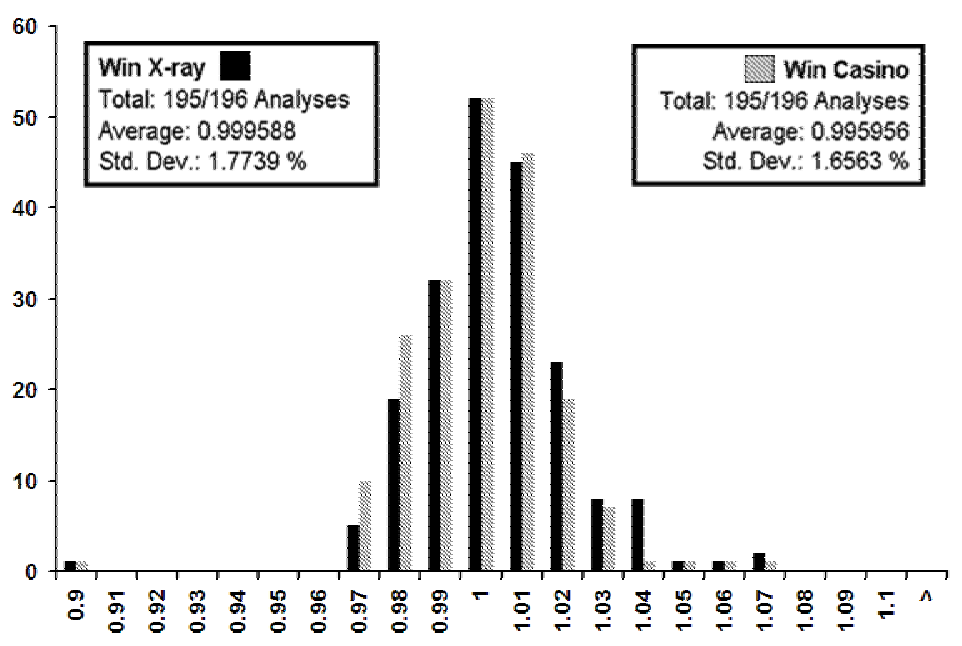

